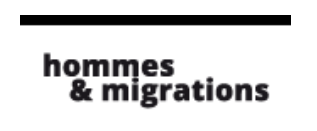

Hommes \& migrations

Revue française de référence sur les dynamiques

migratoires

1305 | 2014

L'exil chilien en France

\title{
Douleur de la séparation et quête de retrouvailles dans la poésie chilienne écrite en exil (1973-1990)
}

\section{Benoît Santini}

\section{(2) OpenEdition}

\section{Journals}

\section{Édition électronique}

URL : http://journals.openedition.org/hommesmigrations/2740

DOI : 10.4000/hommesmigrations. 2740

ISSN : 2262-3353

\section{Éditeur}

Musée national de l'histoire de l'immigration

\section{Édition imprimée}

Date de publication : 1 janvier 2014

Pagination : 135-142

ISBN : 978-2919-040261

ISSN : $1142-852 X$

\section{Référence électronique}

Benoît Santini, « Douleur de la séparation et quête de retrouvailles dans la poésie chilienne écrite en exil (1973-1990) », Hommes \& migrations [En ligne], 1305 | 2014, mis en ligne le 01 janvier 2017, consulté le 21 avril 2019. URL : http://journals.openedition.org/hommesmigrations/2740 ; DOI : 10.4000/hommesmigrations.2740 


\title{
DOULEUR DE LA SÉPARATION ET QUÊTE DE RETROUVAILLES DANS LA POÉSIE CHILIENNE ÉCRITE EN EXIL (1973-1990)
}

par BENOÎT SANTINI, maître de conférences (Littérature et civilisation latino-américaines), université du Littoral-Côte d'Opale, laboratoire H.L.L.I.

\author{
Les exilés chiliens ont produit de nombreuses œuvres poétiques \\ dont la force répond à la violence qui a provoqué leur départ \\ du Chili. Fortes et exaltées, ces œuvres sont emplies de nostalgie \\ pour une patrie que les poètes chiliens en exil doutent de ne \\ jamais revoir. Dans leurs œuvres, ils mettent en scène leur quête \\ d'identité, leur errance et la présence paradoxale de leur \\ pays lointain. Coincés dans l'entre-deux de l'absence, ces poètes \\ conservent intact leur amour du Chili par-delà les aléas de \\ I'histoire.
}

À partir de 1973, de nombreux artistes, parmi eux des poètes, quittent le Chili suite au coup d'État de Pinochet et la mise en place par la junte d'un féroce système répressif. Les poètes chiliens de la diaspora, séparés de leurs racines, proposent, par exemple, un traitement de la thématique de l'exil dans leur production depuis létranger. Le sentiment d'abandon et de solitude de ces auteurs exilés est perceptible dans les poèmes qu'ils créent depuis l'ailleurs. La situation "entre-deux" du poète exilé l'amène à concilier le lien qui l'unit à sa terre et le nouveau lien qui se crée avec le pays d'accueil. Ces poètes peuvent s'exprimer dans des revues publiées hors du Chili, comme Araucaria de Chile qui "a édité à Madrid, depuis 1978, un total de 48 excellents numéros contenant une création abondante, une critique littéraire rigoureuse, des essais et analyses politiques ${ }^{1 "}$, ou encore Literatura chilena en el exilio, publiée en Californie dès 1977, avant de devenir en 1981 lors de son transfert à Madrid Literatura chilena, creación y crítica, mais aussi Lar fondée à Madrid par Omar Lara, comprenant 3 numéros (entre 1981 et 1983). Enfin, le numéro 12 de Ventanal. Revista de creación y crítica ("Muestra de poesía chilena actual", 1987) propose des poèmes souvent inédits d'auteurs chiliens exilés. Des textes subver- 
rique latine en avril 1977), Los poetas chilenos luchan contra el fascismo (Berlin, 1977), Chile: poesía de la resistencia y del exilio (Barcelone, 1978), Chile poesías de las cárceles y del destierro (Madrid, 1978). La production culturelle depuis l'exil est donc riche et plurielle, car "La poésie est un outil de dénonciation des expériences des Chiliens de l'exill". Nous souhaitons nous demander par quels procédés scripturaux les poètes de l'exil sélectionnés dans cet article chantent un déracinement à multiples facettes, les pays d'accueil étant pluriels, et montrer que "les écrivains exilés - et notamment les poètes des années 1970 et 1980 - ont, eux, vécu cette expérience comme un apprentissage authentique de survivance ${ }^{4 "}$.

\section{L'ici et l'ailleurs}

Selon Dominique Berthet, "l'ailleurs n'existe que dans sa relation à l'ici. Si l'ici est par définition l'endroit où l'on est, l'ailleurs est l'en dehors, l'autre part proche ou lointain ${ }^{5 "}$. Dans la poésie chilienne écrite en exil, l'ici se déplace du Chili vers le lieu d'accueil, et l'ailleurs devient paradoxalement le pays d'origine dont le moi poétique est éloigné. Omar Lara

Les poètes chiliens luttent contre le fascisme (c) D.R.

sifs sont ainsi diffusés depuis l'étranger où s'organise une lutte contre la dictature car "en exil, les poètes s'associent à la campagne de dénonciation de la violation des droits de l'homme ${ }^{2 "}$. Des anthologies de poésie voient également le jour, incluant des poètes de l'exil : 10 poetas chilenos en París (édition artisanale publiée par le Centre France Amé-
- exilé au Pérou puis en Roumanie entre 1974 et 1981, avant de s'installer en Espagne - détourne dans le bref poème "Aquí o en la quebrada del aji" (1977, dans Revista chilena del exilio) le proverbe chilien "Aquí y en la quebrada del ajî" (qui signifie qu'une chose est valable en tous lieux). La conjonction "y" ("et") devient "o" ("ou"), la voix lyrique, en raison de son exil, se trouvant désormais "ici" (au Pérou) et non plus au Chili symbolisé par la localité "la quebrada del aji”" de la région de Valparaíso : "Vagando sin sentido por las calles de Lima" " Errant

2. Jorge Montealegre, “Chile, historia de poetas", in Eva Goldschmidt Myman (dir.), Antología. Los poetas y el General. Voces de oposición en Chile bajo Augusto Pinochet 1973-1989, Santiago du Chili, LOM Ediciones, éd. bilingue, décembre 2002, p. 20.

3. Nicolas Prognon, "Producción cultural de los exilados chilenos en Francia: la construcción de una indispensable memoria de la Unidad Popular para la democracia chilena", in Laetitia Boussard, Benoît Santini (dir.), Chile en el siglo xxı: ¿nuevos recorridos artísticos, nuevos caminos históricos?, Santiago du Chili, Mago Editores, Piso Diez Ediciones, 2013, p. 76. 4. Adriana Castillo de Berchenko, “Les éditions bilingues GrilloM. Diffusion de la poésie chilienne dans le Paris des années 1980", in Cahiers d'études romanes, $n^{\circ}$ 14, vol. 2, 2005, p. 287. 5. Dominique Berthet, “Avant-propos”, in Dominique Berthet (dir.), Visions de l'ailleurs, Paris, L'Harmattan, 2009, p. 9. 
sans direction précise dans les rues de Lima") ${ }^{6}$. Le moi fait allusion à une errance absurde dans une ville étrange et étrangère, "sin sentido" renvoyant à la fois à l'absence de signification et de direction, et à ce "aquî" s'oppose donc l'ailleurs, le "là-bas" que Sergio Macías (exilé dès 1973 au Mexique, en Allemagne puis à Madrid) chante dans l'un des poèmes de Memoria del exilio (1985, Madrid) : "Mi patria es el Sur. / Y hacia allá voy como los peces devotos. / No soy más que un enjuncador de la luna" "Ma patrie est le Sud. / Et je vais là-bas comme les poissons dévots. I Je ne suis rien d'autre qu'un rempailleur de la lune $\left.{ }^{7 \prime}\right)$. À travers des images animales et astrales, le moi exprime un mouvement horizontal et vertical, un retour aux sources, élabore des constructions oniriques par la force de la pensée ("rempailleur de la lune") qui lui permettent de supporter l'absence de la terre aimée. Il revendique donc son appartenance à une zone géographique ("el Sur"), effectuant une claire dissociation entre terre d'origine aimée et terre d'accueil où il se voit contraint de résider.

\section{Nostalgie d'un espace et d'un passé}

Les poètes expriment dans une énonciation tourmentée la nostalgie d'un espace et d'un passé perdus : dans Memoria del exilio, la voix chante à la première personne du singulier l'espace naturel de l'Araucanie, leitmotiv lancinant: "Vuelvo a la Araucanía" ("Je retourne en Araucanie"), "las praderas de la Araucanía" ("les prairies de l'Araucanie"), "Subiré a la cima de la Araucanía" ("Je monterai au sommet de l'Araucanie") $)^{8}$. Le mouvement est suggéré par les verbes "volver" et "subir" ("revenir" et "monter"), l'un utopique, car employé au présent de l'indicatif, alors que le retour au Chili n'est pas d'actualité lors du présent de l'énonciation, l'autre prospectif (futur). Cette nostalgie s'accompagne donc d'une forme d'espoir et de confiance en un avenir de retrouvailles. Ce manque se confirme lorsque la voix lyrique mentionne le fleuve d'Araucanie ("río Donguil"), ainsi que les forêts de la province mapuche de Cautín dans cette même région : "fatalidad de no encontrarse en la armonía de los bos-/ques de Cautín ("fatalité de ne pas se trouver dans l'harmonie des bois / de (autín"9). La séparation semble marquée du sceau du destin tragique, la négation souligne la privation dont souffre le sujet poétique ("no encontrarse") et au fleuve Donguil répond le flot des souvenirs, auxquels le moi fait souvent allusion par l'emploi récurrent du vocable "memoria".

\section{Rôle du souvenir}

Le moi d'Omar Lara, pour sa part, par le biais lui aussi du souvenir, se remémore depuis Lima en janvier 1974 - comme cela est indiqué à la fin du poème - le fleuve Valdivia dans le poème "Hablo de Luis Oyarzún, del río Valdivia, etc.", hommage à l'écrivain et académicien Luis Oyarzún. Il cite "la ondulante cola del río Valdivia" ("l'ondulante queue du fleuve Valdivia"), et ajoute: "De ese río viene un susurro de agua y un susurro / de palabras ; / el golpeteo nervioso, calculado / de las embarcaciones" (De ce fleuve vient un chuchotement d'eau et un chuchotement / de mots ; / le clapotis nerveux, calculé / des embarcations" $)^{10}$. Les sens sont sollicités (vue, ouïe) depuis l'exil dans une tentative de reméLa situation "entre-deux" du poète exilé l'amène à concilier le lien qui l'unit à sa terre et le nouveau lien qui se crée avec le pays d'accueil. moration et de préservation du souvenir de lieux familiers. Cette nostalgie s'applique aussi à un passé révolu et prend la forme d'un hôtel dans "Hotel de las nostalgias" d'Óscar Hahn, exilé dès 1974 aux États-Unis : "Nosotros / los adolescentes de los años 50 / los del jopo en la frente y el pucho en la comisura", "dónde estamos ahora I que la vida es de minutos nada más / en qué campo de concentración / asilados en qué embajada / en qué 
país desterrados 11" "Nous / les adolescents des années 50 / ceux avec la clope au coin des lèvres", "où sommesnous maintenant / que la vie est faite de minutes rien de plus / dans quel camp de concentration / réfugiés dans quelle ambassade / dans quel pays exilés"). Dans une énonciation à la première personne du pluriel, le pays d'accueil est assimilé à un hôtel, lieu de passage avant un retour espéré au Chili. Ce poème accusateur, hommage intertextuel au rock Hotel Heartbreak d'Elvis Presley, dont les adolescents des années 1950 au Chili étaient friands, est écrit et publié depuis l'exil (les États-Unis, l'évocation à la chanson d'Elvis renvoyant à un espace géographique nord-américain,) dans une revue de la diaspora ; il reflète le regret d'un
Les voix sont scindées, souffrantes, à la limite de la schizophrénie en raison de l'éloignement et des manques qui les empêchent parfois de nommer l'altérité, la ville ou le pays d'accueil. passé d'insouciance et d'une jeunesse révolue, mais aussi du Chili lointain.

Parfois, c'est le souvenir de l'exil lui-même qui est au cœur des poèmes. Le moi du poème autobiographique en français de Mauricio Electorat, "Panne sèche ou pourquoi je n'ai pas écrit un seul poème depuis que je suis à Paris" (1990), preuve de l'influence de son séjour français sur sa production poétique, explique : “C'était en 1987 / Je vivais à Barcelone sans le sou / Même si jétais lecteur chez Balcells (...) Je fis un détour par Sète, / Je pris un coup de vent à Narbonne / Et deux mois après jétais installé à Paris / Comme il convient, dans une chambre de bonne $e^{12}$." Les références spatio-temporelles et la précarité de la situation du moi renvoient à une douloureuse réalité vécue par le poète lui-même, lequel est sermonné par la voix de Gustavo Mujica, l'incitant à publier ses poèmes. Les temps du passé plongent la voix lyrique au début de son exil, période à laquelle l'adaptation en terre étrangère marquée par l'errance s'avère difficile.

\section{La négation, mécanisme scriptural}

Certains poètes utilisent par ailleurs comme trait distinctif la négation, suggérant la privation, l'absence, la perte et l'abandon. Patricia Jérez, exilée à Paris en 1973, y publie Jaque (éditions GrilloM, 1985) après la sortie en 1983 à Madrid (dans la revue littéraire $L A R$ ) de Enroque. Le poème "Exilio", dans 10 poetas chilenos en París, se construit sur la récurrence sémantique et anaphorique de la négation "Nadie" : "Nadie nos vio pasar", "nadie nos vio correr", "nadie nos vio partir", "nadie nos oyó cantar" ("Personne ne nous a vus passer", "personne ne nous a vus courir", "personne ne nous a vus partir", "personne ne nous a entendus chanter" $)^{13}$. Cette fuite discrète, précipitée ("correr"), invisible et inaudible, reflète le départ du Chili pour des raisons politiques, l'absence et le manque subséquents que l'on découvre aussi chez Gonzalo Santelices qui, avec ses parents, s'exile en Espagne à 16 ans en 1977 et y publie Sueño en la torre (1985), dont l'un des poèmes commence par: "No hallarás las tierras / que dejaron tus padres" ("Tu ne trouveras pas les terres / que tes parents ont laissées") ${ }^{14}$. Un dédoublement s'opère à travers un tutoiement par le biais duquel le moi semble s'adresser à lui-même. Ces "terres" abandonnées s'apparenteraient au Chili, espace inaccessible étant donné l'absence et l'éloignement (la négation est très éloquente). La négation exprime également le sentiment de rejet éprouvé par les sujets lyriques déracinés. Dans le poème anonyme "Primer día de exilio" ("Premier jour d'exil"), paru dans Chile poesías de las cárceles y del destierro, on lit: "París es helado, muy frío. / No es para el que llega", "París es caro, / no es para el pobre", "París no es para mí / ni yo para él" ("Paris est glacé, très froid. / Il n'est pas fait pour celui qui arrive", "Paris est cher, / il n'est 
Les poètes et le Général, LOM Ediciones (c) D.R.

pas fait pour le pauvre", "Paris n'est pas fait pour moi / ni moi pour lui") ${ }^{15}$, la rigueur du climat parisien déclenche un discours scindé envahi de négations, confirmant la difficulté d'adaptation du moi exilé dans la capitale française.

\section{Manque affectif}

Le manque est géographique et affectif, la voix étant parfois éloignée de ses proches comme dans "Aquí o en la quebrada del aji" : "Amor mío yo me junto contigo donde sea / Me haces una falta de carajo" ("Mon amour je te rejoins n'importe où / tu me manques vachement"16). L'apostrophe déchi- rante à l'être aimé, sans doute restée au Chili, creuse l'abîme dans lequel est plongée la voix. La perte est enfin linguistique, puisque le moi de "Bonne année" (1983, revue LAR), de José María Memet, exilé à Paris entre 1981 et 1985, adopte une langue étrangère : "En la solitaria mesa donde ceno / me doy cuenta - entre risas / y saludos en francés - que / me han quitado los derechos a comer / el pan en castellano" "“̀̀ la table solitaire où je dîne / je me rends compte - parmi les rires / et les salutations en français - que / l'on m'a ôté le droit de manger / le pain en castillan") ${ }^{17}$. Le manque se matérialise par le vide verbal d'un poème de Aisla (Paris, 1984) de Felipe Tupper, constitué de vers brefs, nominaux, dont l'énonciation hachée, succincte, va de pair avec les carences du moi séparé de son Chili et exilé aux Baléares: "Formentera / Humedad / Desalojamiento / Distancia" ("Formentera / Humidité / Délogement / Distance") $)^{18}$. La difficulté d'adaptation est davantage climatique ("Humedad") que linguistique, malgré la présence d'une autre langue, le catalan, aux Baléares.

\section{Souffrance et altérité}

Les voix sont scindées, souffrantes, à la limite de la schizophrénie en raison de léloignement et des manques qui les empêchent parfois de nommer l'altérité, la ville ou le pays d'accueil. Gustavo Mujica dans "Sintonía" (revue LAR, 1983), écrit : "En el Metro de la ciudad sombra musiquean algunos músicos, / Músicos japoneses cantaban el otro día, en el Metro Odeón" ("Dans le métro de la ville sombre quelques musiciens jouent de la musique, / Des musiciens japonais chantaient l'autre jour, au métro Odéon") ${ }^{19}$. Même si le nom de Paris apparaît dans ce poème, l'expression "ciudad sombra" renverse la célèbre périphrase "Ville lumière", faisant de la capitale un lieu téné- 
breux, reflet de l'esprit torturé du moi plongé dans les affres de ce que Julia Kristeva appelle une "troublante altérité" ${ }^{20}$, laquelle se démultiplie par l'évocation de musiciens japonais, eux aussi déracinés, dans un métro parisien. Ce poème empreint d'humour et construit sur des affirmations se succédant sous la forme d'anadiploses témoigne du choc culturel vécu par une voix coupée de son monde. Waldo Rojas, dans "Verano de exilio", composé à Cannes et Paris en 1974, écrit: "Recrudece el verano en la tierra del Torso Mutilado / mientras tuerce mi exilio otra vuelta de niebla sobre / el país-naufragio" "'L'été s'intensifie sur la terre du Torse Mutilé / pendant qu'un nouveau retour de brouillard sur le paysnaufrage tord mon exil") ${ }^{21}$. Les images de fragmentation, de déformation, d'obscurité créent un discours torturé, qui ne permet pas de nommer les pays d'accueil et d'origine : le "Torse Mutile" serait le Chili, effectuant un chemin de croix dès 1973, supplicié par les militaires et Pinochet et le "pays-naufrage" représenterait la France où s'échouent les exilés. Ces images expressives invitent le lecteur à recomposer un discours meurtri et assombri par la rigueur climatique qu'inflige le "brouillard".

\section{Naufrage et errance}

Cette image de naufrage, cause de la douleur du moi, est fréquente dans les poèmes de l'exil. Adriana Castillo de Berchenko écrit que la métaphore du naufrage dans la poésie chilienne évoque "un moi égaré, errant dans un monde inconnu, hostile et menaçant" ou encore "l'engloutissement définitif d'une société entière22". Gonzalo Santelices, dans le poème cité plus haut, mentionne les "restos de un antiguo naufragio en la piel" ("les restes d'un ancien naufrage dans la peau" ${ }^{23}$ en référence aux parents

"La poésie chilienne de la résistance et de l'exil." (c) D.R.

du moi lyrique, portant en eux les souvenirs du Chili brutalisé et eux-mêmes expulsés de leur terre. Patricia Jérez, dans "Exilio", écrit : "Solos, con una palmera y un sol naufragado / lanzaremos la última botella con el último mensaje" ("Seuls, avec un palmier et un soleil naufragé / nous lancerons la dernière bouteille avec le dernier message" ${ }^{24}$. Dans "Visiones de Colón" (de Un buey sobre mi lengua, 1987) de Mauricio Electorat, la voix poétique utilise la métaphore filée du naufrage, se comparant à "un búfalo sobre la gruesa arena" ("un buffle sur le sable épais"), mentionnant "las podridas maderas" ("les bois pourris") et le "final de la playa, donde naufraga la terraza de mármol" " "extrémité de la plage, où naufrage la terrasse de marbre") ${ }^{25}$. Solitude, vie échouée, décomposition de 
la frêle embarcation sur laquelle dérivent les voix poétiques traduisent la précarité et la souffrance de ces voix forcées au déplacement à bord d'un nouveau radeau de la méduse.

L'errance est elle aussi au cœur de l'énonciation de ces poèmes : elle est géographique, comme le montrent les sujets lyriques devenus homo viator et mulier peregrina moyennant l'emploi des verbes "vagar" (Omar Lara $\left.{ }^{26}\right)$, "pasar" (Patricia Jérez), "viajar" $\left(\right.$ Memet ${ }^{27}$ ), "recorrer el mundo" (Gustavo Mujica ${ }^{28}$ ), les vocables et expressions "hombre errante", "peregrino, viajero, caminante" (Sergio Macias) ${ }^{29}$. L'errance est aussi celle du sens fuyant, échappant parfois au lecteur : les images employées par Mauricio Electorat, l'imprécision de certains vers chez Omar Lara, la disposition typographique particulière du poème sans titre de Gustavo Mujica, construit sur la récurrence lexicale de "ninguna parte" va de pair avec l'errance physique du moi en mouvement perpétuel dans des poèmes où prédomine l'esthétique du déplacement.

\section{Perte d'identité et dédoublement}

Ce mouvement engendre scission et fracture du moi, confirmant les propos de Nicolas Bouvier: "C'est le voyage qui vous fait, ou vous défait ${ }^{30}$." Dans certains cas, le moi poétique ne se reconnaît pas, comme le corrobore l'image du miroir chez José María Memet ("al mirar casualmente a la ventana / observo una pupilas / donde existe desarraigo, / mírolas bien y al hacerlo / me doy cuenta que son mías" : "en regardant par hasard vers la fenêtre / jobserve des pupilles / dans lesquelles existe de l'exil, / je les regarde bien et en le faisant / je me rends compte que ce sont les miennes") 31 et chez Sergio Macias ("Me miro en el espejo del día (...). Me observo como un ex-/traño. / No me encuentro. El espejo se multiplica"; "Je me regarde dans le miroir du jour (...). Je m'observe comme un é/tranger. I Je ne me retrouve pas. Le miroir se multi- plie") $)^{32}$ et le sujet poétique dédoublé perd son identité. Michel Onfray écrit: "Que reste-t-il de mon identité dès la suppression des attaches sociales, communautaires, tribales, quand je me retrouve seul, ou presque, dans un environnement sinon hostile, du moins inquiétant, troublant, angoissant ${ }^{33}$ ?" Ces sujets poétiques démontrent cette perte d'“attaches" et leur difficile intégration dans un nouvel univers "angoissant". Le dédoublement s'accompagne d'une perte de repères : “Dónde estoy ?” (“Où suis-je ?”), se demande la voix poétique de Sergio Macias ${ }^{34}$, "dónde estamos ahora" ("où sommes-nous désormais ?"), s'interroge celle d'Óscar Hahn ${ }^{35}$. L'interrogation devient l'un des traits distinctifs de l'écriture poétique de la diaspora, preuve de la désorientation de sujets propulsés dans un monde qui n'est pas le leur et qui devient souvent un "non-lieu".

\section{Se reconstruire par l'écriture}

Face au déracinement, l'écriture devient survie et possibilité de reconstruction. Les poètes exilés dénoncent par le non-dit ou sans détour la situation politique de leur pays et se libèrent d'un poids par le langage, l'humour corrosif, l'irrévérence. Dans Face au déracinement, La Ciudad (1979) de Gon- l'écriture devient survie et zalo Millán, exilé au Canada possibilité de reconstruction après le coup d'État, le moi, Les poètes exilés dénoncent par l'usage de propositions parlenon-ditousans brèves, répétitives, apparem- détour la situation politique ment neutres, montre du deleur pays et selibèrent doigt les privations infligées d'un poids par le langage aux Chiliens, entre autres l'humour corrosif, l'irrévérence la confiscation du pays et l'exil contraint: "Desapoderaron sindicatos. / Desapropiaron industrias. / Desampararon a los huérfanos. / Desarraigaron compatriotas" ("Ils ont dépossédé les syndicats. I Ils ont exproprié les industries. / Il ont délaissé les orphelins. I Il ont déraciné des 
compatriotes" $)^{36}$. Le privatif "des-", accusateur, retire aux Chiliens leurs acquis et possessions. La troisième personne du pluriel, sans sujet clairement exprimé, renvoie par l'implicite à l'autoritarisme du général et de ses militaires. Nombre de poètes de l'exil cités dans cet article utilisent la constellation sémantique de l'exil : "exilio" (Waldo Rojas ${ }^{37}$ ), "diáspora" (Gustavo Mujica ${ }^{38}$ ), "desterrados" (Óscar $\mathrm{Hahn}^{39}$ ), "exilio", "desterrado", "éxodo", "desarraigo" $\left(\right.$ Sergio Macias $\left.^{40}\right)$. Les poètes parviennent aussi parfois à nommer le lieu d'exil et à revendiquer la présence dans celui-ci : Waldo Rojas dans "Verano de exilio"1" date son poème et en indique le lieu de création (“Cannes-París-julio 1974”); Omar Lara fait de même dans "Hablo de Luis Oyarzún..." ("Lima, enero, 1974"2") et dans "Aquí o en la quebrada del aj44", il évoque l'errance dans les "calles de Lima". Mauricio Electorat, dans "Visiones de Colón", cite les "Baños de mar Francia" et "Hotel de Francia" 4 , le pays d'accueil devenant un territoire aux dimensions imprécises, exigu comme une chambre d'hôtel ou vaste comme la mer. Le déracinement et le choc culturel vécus dans un pays inconnu sont des traumatismes que seule la langue poétique peut calmer.

Les poèmes cités, aperçu d'une riche production, démontrent que le lieu d'origine des poètes exilés continue "à 'être présent en dépit de l'absence ${ }^{45}$ " et, en même temps, ils s'approprient leur nouvel espace, même si l'adaptation s'avère compliquée. L'intégration requiert en particulier un apprentissage de la langue du pays d'accueil, comme le démontrent Memet et Electorat. Toutefois, l'importance du souvenir permet aux voix poétiques et à leurs créateurs de garder le lien avec la terre d'où ils proviennent. Sergio Macias, par exemple, emploie à satiété les termes de "recuerdo", "memoria", jusque dans son titre (Memoria del exilio). Il s'agit de lutter contre un double oubli : celui d'un pays avec lequel ils ne sont plus directement en contact et celui d'événements traumatisants dans lesquels ils se retrouvent ou se sont retrouvés immergés (coup d’État, répression, exil). Ainsi, la dénonciation, souvent effectuée par le non-dit ou la suggestion, revient sur les conditions de l'exil, mais aussi sur les événements vécus au Chili. Il s'agit de rester ancré dans une réalité chilienne malgré l'éloignement.

Dans ces poèmes, pied de nez à la dictature qui récupère les symboles patriotiques en les déviant de leur fonction première, les voix lyriques redonnent au mot "patrie" sa vraie valeur : Waldo Rojas, Gustavo Mujica, Sergio Macías ${ }^{46}$ utilisent ce terme, menant depuis l'étranger un combat poétique contre la junte et coopérant à distance, comme tous les poètes cités, avec leurs compatriotes restés au Chili.

Il serait réducteur de limiter ces poèmes à une reproduction mimétique du réel. Ils sont également l'occasion pour leurs auteurs de mettre en œuvre des jeux verbaux, de faire montre du plaisir qu'ils ont à manier les mots. Les vers nominaux chez Sergio Macías expriment sans détours les états d'âme du sujet lyrique et la difficulté d'exprimer ses angoisses. Les vers brefs ou longs, les récurrences lexicales et les échos contribuent à transmettre le message véhiculé par ces textes de la diaspora chilienne. Les auteurs, grâce à leur agilité verbale, font de leurs poèmes des textes cathartiques, la création étant salutaire et leur permettant d'affronter les contingences dans lesquelles ils vivent, dans l'espoir de retrouver un jour un Chili démocratique. 